\title{
Misorientation Gradients by EBSD in Hot and Cold Deformed Alloys
}

\author{
T. Zhai*, X.P. Jiang**, J.H. Xu***, Q. Zeng*, W. Wen*
}

* Department of Chemical and Materials Engineering, University of Kentucky, 177 F. Paul

Anderson Tower, Lexington, KY 40506

** Now with Shaw Group, 1430 Enclave Pkwy, Houston, TX 77077

*** Now with Smith International, Houston, TX

Characterization of plastic strain or residual stresses on a microscopic scale is important for understanding property-microstructure-processing relationships in alloys. Local elatisc strain can be measured using a zone axis shift method by EBSD [1], while the global plastic strain could be determined by measuring the average misorientation over many grains with EBSD [2]. In this work, EBSD was used to characterize misorientation gradients within individual grains in a hot extruded AA2099 Al-Li alloy and a cold deformed 50Mo-50Re alloy. New generation AA2099 Al-Li alloys possesses superior high cycle fatigue properties and high strength, e.g., their fatigue strength is over $90 \% \sigma_{\mathrm{y}}$, compared to about $45 \% \sigma_{\mathrm{y}}$ for most conventional high strength $\mathrm{Al}$ alloys [3]. Their fatigue strengening mechanisms still need to be fully understood. Fig. 1 shows an EBSD orientation map of the alloy which had almost a layered grain structure. Within each grain, the crystallographic orientation changed gradually in the direction perpendicular to the extrusion direction (Figs. 1(b) \& (c)), but remained almost constent along the extrusion direction (Fig. 1(d)). The gradual change in orientation should be related to the change of the degree of plastic flow in the direction perpendicular to the extrusion direction in each grain in the alloy during hot extrusion. Since the alloy could not be recrystallized because of the addition of $\mathrm{Zr}$, the gradual change in misorientation within each grain was in the form of a series of subgrains which changed their orientations gradually in this direction. These subgrains were formed during the recovery of the alloy in cooling or solid solution treatment. This work demonstrated that the alloy behaved like a viscous material during hot extrusion, thereby causing a gradual increase in shear strain towards the center in the direction perpendicular to the extrusion dirction within each grain.

Fig. 2(a) shows a crack formed at a grain boundary triple junction during tensile deformation in a 50Mo-50Re alloy. The surface of the alloy deformed at a strain rate of $10^{-1} \mathrm{~s}^{-1}$ in tension followed by etching is shown in Fig. 2(b) and its EBSD orientation mapping is given in Fig. 2(c) where the maximum misorientation occurs at a grain boundary. The misorientation and its gradient profiles along line AB in Fig. 2(c) are illustrated in Fig. 2(d). It can be seen that the maximum misorientation occurred at the grain boundary, but the maximum misorientation gradient was about $2.2 \mu \mathrm{m}$ away from the boundary. This result explained that strain concentration was responsible for the observed crack initiation at grain boundary triple junctions in the 50Mo-50Re alloy. The maximum plastic strain took place about $2.2 \mu \mathrm{m}$ away from grain boundaries during tensile deformation in this alloy.

References

[1] K.Z. Troust, P. van der Sluis, D.J. Gravesteijn, Applied Physics Letter, 62, 10 (1993), 1110-1112. [2] M. Kamaya, A.J. Wilkinson, J.M. Titchmarsh, Acta Mat., 54 (2006), 539-548.

[3] X.P. Jiang, PhD Thesis, University of Kentucky, 2007.

[4] J.H. Xu, E.W. Kenik, T. Zhai, Phil Mag. A, 88(2008), 1543-1553. 

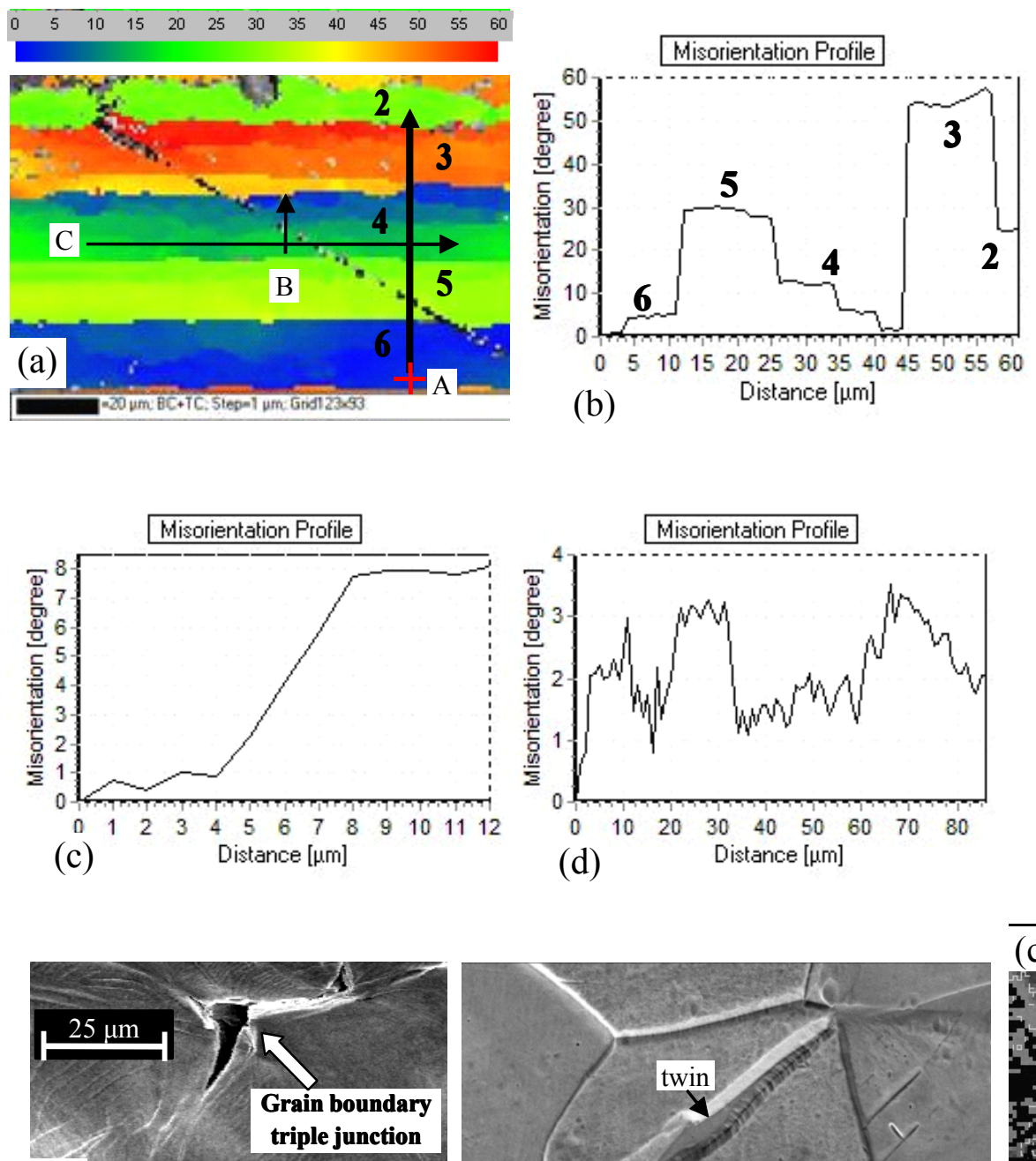

(a)
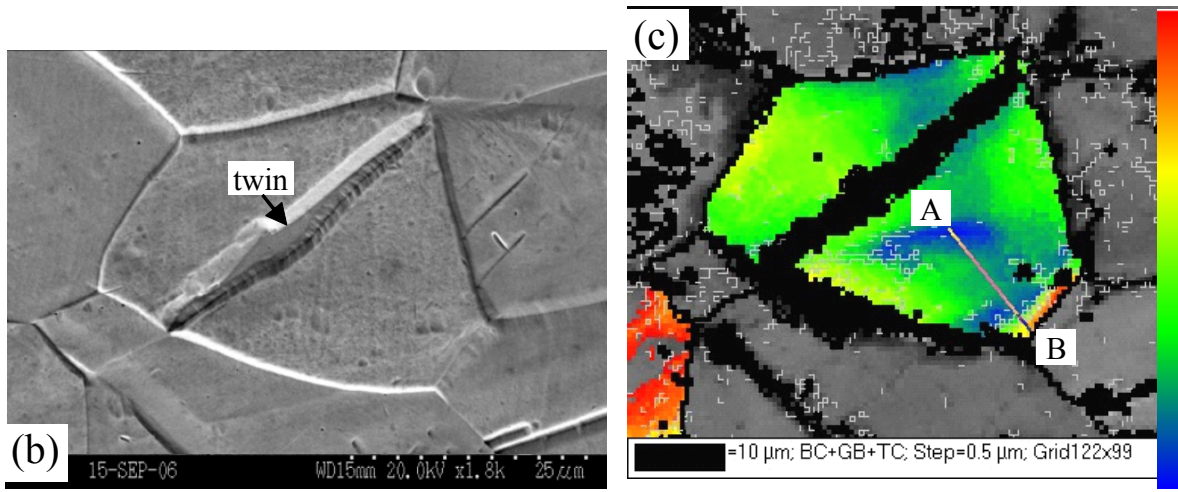

Fig. 1 (a) Orientation mapping of an AA2099 AlLi alloy after hot extrusion. (b) misorientation profile along line $\mathrm{A}$ in (a). (c) misorientation profile along line B in (a). (d) misorientation profile along line $\mathrm{C}$ in (a).
(d)

Fig. 2 (a) cracking at a grain boundary triple junction during tensile deformation in a $50 \mathrm{Mo}-50 \mathrm{Re}$ alloy; (b) SEM micrograph of the surface deformed at $10^{-1} \mathrm{~s}^{-1}$ strain rate in tension; (c) EBSD orientation map of the same area as in (b). A large misorientation occurs at a grain boundary. The rainbow color scale from blue to red is equivalent to $18^{\circ}$ of misorientation. (d) The orientation profile and misorientation gradient from point $\mathrm{A}$ to $\mathrm{B}$ in (c). The maximum misorientation, i.e., the maximum strain, occurs at about $2.2 \mu \mathrm{m}$ away from the grain boundary. 